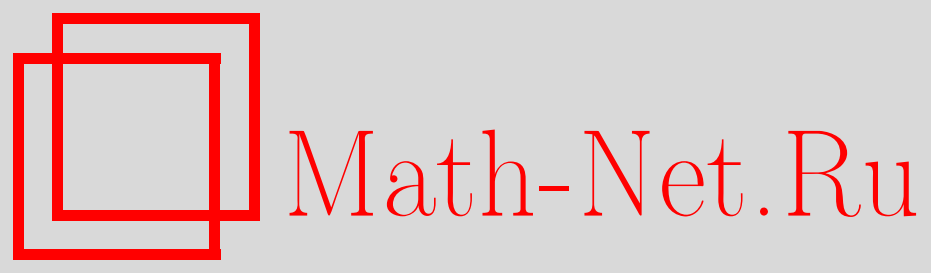

Г. В. Балакин, Линейные псевдобулевы неравенства, Матем. вопр. криптогр., 2010, том 1, выпуск 3, 5-18

DOI: https://doi.org/10.4213/mvk12

Использование Общероссийского математического портала Math-Net.Ru подразумевает, что вы прочитали и согласны с пользовательским соглашением http://www.mathnet.ru/rus/agreement

Параметры загрузки:

IP : 35.174 .16 .151

26 апреля 2023 г., 02:23:08 
УДК 519.212.2

\title{
Линейные псевдобулевы неравенства
}

\author{
Балакин Г. В. \\ Академия криптографии Российской Федерации, Москва
}

Получено 22.IV.2010

В работе рассматриваются случайные и заведомо совместные системы из линейных псевдобулевых неравенств. Найдены оценки вероятности совместности и апостериорные распределения неизвестных.

Ключевые слова: линейные псевдобулевы неравенства

\section{Linear pseudo-Boolean inequalities}

\section{G. V. Balakin}

Academy of Cryptography of Russian Federation, Moscow

\begin{abstract}
Systems of random linear pseudo-Boolean inequalities are considered. Probability of solvability is estimated. Conditional distributions of solutions of solvable systems are investigated.
\end{abstract}

Key words: linear pseudo-Boolean inequalities, solvability probability

Citation: Mathematical Aspects of Cryptography, 2010, vol. 1, no. 3, pp. 5-18 (Russian). 


\section{1. Введение}

Рассмотрим над кольцом целых чисел систему неравенств

$$
a_{i 1} x_{1}+a_{i 2} x_{2}+\ldots+a_{i n} x_{n} \geq b_{i}, \quad i=1, \ldots, t,
$$

с неизвестными $x_{1}, x_{2}, \ldots, x_{n}$, принимающими только два значения: 0 или 1. Заметим, что к системе вида (1) сводятся другие системы линейных неравенств. Действительно, знаки в правой части $<$ и $\leq$ всегда можно поменять на противоположные, а знак > можно заменить на знак $\geq$, если подходящим образом увеличить правую часть неравенства.

По мнению автора, в общем случае труднее всего решать системы неравенств (1), которые удовлетворяют следующим условиям:

1) в каждом неравенстве присутствуют все $n$ неизвестных;

2) каждому неравенству удовлетворяет примерно половина векторов $x=\left(x_{1}, \ldots, x_{n}\right)$ из некоторого множества возможных решений $X \subseteq\{0,1\}^{n} ;$

3) среди коэффициентов в левых частях неравенств (1) нет выделяющихся.

Если система (1) заведомо совместна, то предполагаем, что в истинном решении $x^{0}$ содержится примерно половина нулей и единиц.

Автору пока удалось найти только один класс систем (1), неравенства в которых строго удовлетворяют перечисленным выше условиям. Этот класс систем характеризуется следующими параметрами:

- число неизвестных $n=2 m, m$ - нечётное число;

- все коэффициенты в левых частях неравенств (1) равны 1 или -1;

- $m$ коэффициентов равны 1 и $m$ коэффициентов равны -1 ;

- множество возможных решений $X$ состоит из векторов $x$ нечетного веca;

- $\quad$ все правые части в неравенствах (1) равны 1.

Если система (1) заведомо совместна, то истинное решение $x^{0}$ имеет вес $\left|x^{0}\right|=m$.

Заметим, что в случае, когда $n$ - нечётное число или $m$ - чётное число, то, перебрав определённые значения некоторого числа неизвестных, мы получим в итоге чётное число оставшихся неизвестных и нечётный вес соответствующего им истинного решения. Но при этой процедуре возможны изменения в правой части исходной системы неравенств. 
Следует ещё отметить, что неравенства с коэффициентами, равными 1 или -1 , могут быть преобразованы в неравенства, в которых коэффициенты принимают значения 0 или 1 . Для этого зададим вес вектора $x=\left(x_{1}, \ldots, x_{n}\right)$ :

$$
|x|=x_{1}+x_{2}+\ldots+x_{n}=k,
$$

сложим это равенство с неравенством из системы (1), полученное неравенство разделим на 2 , в итоге имеем неравенство с коэффициентами в левой части, равными 1 и 0 . Обратное преобразование выполняется следующим образом: и итоговое неравенство умножаем на 2 и вычитаем из него заданное равенство. Эту процедуру проводим $(n+1)$ раз при $k=0,1, \ldots, n$, причём случаи $k=0$ и $k=n$ непосредственно проверяются.

\section{2. Случайные системы неравенств}

Пусть коэффициенты $a_{i j}, j=1, \ldots, n, i=1, \ldots, t$, являются реализациями независимых случайных величин $\xi_{i j}, j=1, \ldots, n, i=1, \ldots, t$, принимающих значения +1 и -1 с одинаковой вероятностью $1 / 2$. Выбор правой части не зависит от выбора левой части, причём с вероятностью 1/2 выбирается правая часть вида $\geq 1$ или вида $\leq-1$. Меняем знак у неравенств второго вида и приходим к системе неравенств (1) с $b_{i}=1, i=1, \ldots, t$.

Пусть множество возможных решений $X$ содержит только векторы нечётного веса. Тогда $|X|=2^{n-1}$ и среднее число решений случайной системы неравенств равно $2^{n-1-t}$.

Попытаемся алгебраическим способом, применяя метод выделения и оценки отдельных неизвестных [1], определить факт несовместности системы неравенств либо определить значения отдельных неизвестных. Для этого сложим все $t_{1 i}$ неравенств, в которых коэффициент при $x_{i}$ равен 1 , и $t_{0 i}$ неравенств с коэффициентом при $x_{i}$ равном $-1, t_{1 i}+t_{0 i}=t$. В итоге получим два неравенства следующего вида:

$$
\begin{gathered}
t_{1 i} x_{i}+L_{1 i}+L_{2 i}-L_{3 i}-L_{4 i} \geq t_{1 i}, \\
-t_{0 i} x_{i}+Q_{1 i}-Q_{2 i}+Q_{3 i}-Q_{4 i} \geq t_{0 i}, i=1, \ldots, n .
\end{gathered}
$$

Здесь линейные формы $L_{j i}$ и $Q_{j i}$ имеют неотрицательные коэффициенты и зависят от общих $n_{j i}$ неизвестных, $j=1,2,3,4, \quad n_{1 i}+n_{2 i}+n_{3 i}+n_{4 i}=n-1$. Обозначим $\left|L_{j i}\right|\left(\left|Q_{j i}\right|\right)$ сумму коэффициентов в линейных формах $L_{j i}\left(Q_{j i}\right)$, $j=1,2,3,4, \quad i=1, \ldots, n$.

Для рассматриваемого примера справедливо следующее утверждение. 
Теорема 1. Рассматриваемая случайная система неравенств несовместна, если найдётся такое $i \in\{1,2, \ldots, n\}$, для которого выполняется хотя бы одно из двух условий:

1) $\left|Q_{1 i}\right|+\left|Q_{3 i}\right|<t_{0 i}$;

2) $\left|L_{1 i}\right|+\left|L_{2 i}\right|<t_{1 i}, \quad t_{0 i} \leq\left|Q_{1 i}\right|+\left|Q_{3 i}\right|<2 \cdot t_{0 i}$.

Доказательство. Система из двух неравенств (2) и (3) при $x_{i}=0$ принимает вид

$$
\begin{aligned}
& L_{1 i}+L_{2 i}-L_{3 i}-L_{4 i} \geq t_{1 i}, \\
& Q_{1 i}-Q_{2 i}+Q_{3 i}-Q_{4 i} \geq t_{0 i}
\end{aligned}
$$

и не имеет решений, если справедливо одно из неравенств

$$
\left|L_{1 i}\right|+\left|L_{2 i}\right|<t_{1 i}, \quad\left|Q_{1 i}\right|+\left|Q_{3 i}\right|<t_{0 i} .
$$

Аналогично для $x_{i}=1$ система из неравенств

$$
\begin{array}{r}
L_{1 i}+L_{2 i}-L_{3 i}-L_{4 i} \geq 0, \\
Q_{1 i}-Q_{2 i}+Q_{3 i}-Q_{4 i} \geq 2 t_{0 i}
\end{array}
$$

не имеет решения, если выполняется неравенство

$$
\left|Q_{1 i}\right|+\left|Q_{3 i}\right|<2 t_{0 i} \text {. }
$$

Отсюда получаем утверждение теоремы 1.

Из доказательства теоремы 1 следует, что $x_{i}=0$, если выполняются неравенства

$$
\left|L_{1 i}\right|+\left|L_{2 i}\right| \geq t_{1 i}, \quad t_{0 i} \leq\left|Q_{1 i}\right|+\left|Q_{3 i}\right|<2 t_{0 i},
$$

и $x_{i}=1$, если

$$
\left|L_{1 i}\right|+\left|L_{2 i}\right|<t_{1 i}, \quad\left|Q_{1 i}\right|+\left|Q_{3 i}\right| \geq 2 t_{0 i} .
$$

В случае нахождения значений отдельных неизвестных поступаем следующим образом. Подставляем значения этих неизвестных в неравенства (2), (3). Это приведёт к изменению некоторых линейных форм и правых частей неравенств (2), (3) и соответственно линейных форм и правых частей неравенств, приведённых в теореме 1 . Далее применяем теорему 1 к изменённой системе, находим значения ещё нескольких неизвестных либо устанавливаем факт несовместности системы неравенств, либо ничего не находим. В первом случае процесс продолжается, во втором случае он останавливается, а в третьем случае, по-видимому, следует перейти к подбору значений для нескольких неизвестных.

Теорема 1 верна и для произвольной системы неравенств (1). Для этого достаточно заменить $t_{1 i}\left(t_{0 i}\right)$ суммой правых частей уравнений, в которых коэффициент при $x_{i}$ равен неотрицательному (неположительному) числу. Но при этом коэффициент при $x_{i}$ может отличаться от значения правой части. 
Исследуем поведение вероятностей выполнения неравенств (4) и (5) и неравенств из условий теоремы 1 . Пусть $n, t \rightarrow \infty$. Очевидно, что в этом случае $t_{1 i} \approx t_{0 i} \approx t / 2, \quad n_{1 i} \approx n_{2 i} \approx n_{3 i} \approx n_{4 i} \approx n / 4$ и предельным распределением случайных величин $\eta_{1 i}, \eta_{2 i}, v_{1 i}, v_{3 i}$, реализациями которых являются $\left|L_{1 i}\right|,\left|L_{2 i}\right|,\left|Q_{1 i}\right|,\left|Q_{3 i}\right|$ соответственно, после соответствующей их центрировки и нормировки является нормальный закон с параметрами $(0,1)$. При любых фиксированных $i, t_{1 i}, t_{0 i}, n_{1 i}, n_{2 i}, n_{3 i}, n_{4 i}$ рассматриваемые четыре случайные величины независимы в совокупности.

Рассмотрим случайную величину

$$
\eta_{i}=\sum_{j=1}^{T} \xi_{j i}
$$

Легко видеть, что при $T \rightarrow \infty$ предельным распределением случайной величины $\eta_{i}$ является нормальное распределение с параметрами

$$
\mathbf{E} \eta_{i}=0, \quad \mathbf{D} \eta_{i}=T,
$$

поэтому

$$
\begin{gathered}
m_{i}=\mathbf{E}\left|\eta_{i}\right| / \sqrt{\mathbf{D}\left(\eta_{i}\right)} \sim 2 \cdot \frac{1}{\sqrt{2 \pi}} \int_{0}^{\infty} z e^{-z^{2} / 2} d z=\sqrt{\frac{2}{\pi}}, \\
\mathbf{E}\left(\frac{\eta_{i}}{\sqrt{T}}\right)^{2} \sim 1, \quad \sigma_{i}^{2}=\mathbf{D}\left|\frac{\eta_{i}}{\sqrt{T}}\right| \sim 1-\frac{2}{\pi} .
\end{gathered}
$$

При $T=t_{1 i}$ случайная величина $\eta_{1 i}$ является суммой $n_{1 i}$ независимых случайных величин, у которых математическое ожидание $m_{i 1}$ эквивалентно $\sqrt{\frac{2}{\pi} t_{1 i}}$, а дисперсия $\sigma_{i 1}^{2}$ эквивалентна $t_{1 i}\left(\frac{\pi-2}{\pi}\right)$. Следовательно, распределение случайной величины

$$
\left(\eta_{1 i}-n_{1 i} \sqrt{\frac{2}{\pi} t_{1 i}}\right) / \sqrt{n_{1 i} t_{1 i}\left(\frac{\pi-2}{\pi}\right)}
$$

при $n_{1 i}, t_{1 i} \rightarrow \infty$ стремится к нормальному закону с параметрами $(0,1)$. То же самое можно сказать о случайных величинах $\eta_{2 i}, v_{1 i}, v_{3 i}$. Для $T=t_{0 i}$ применяем обозначения $m_{i 0}, \sigma_{i 0}$.

Приведём конкретные выражения для трёх первых моментов случайной величины $\left|\eta_{i}\right|$ при нечётном $T$. Так как

$$
p_{j}=\mathbf{P}\left\{\left|\eta_{i}\right|=j\right\}=C_{T}^{(T+j) / 2} / 2^{T-1}, \quad j \in I=\{1,3,5, \ldots, T\},
$$


то очевидно, что

$$
\begin{gathered}
\mathbf{E}\left|\eta_{i}\right|=\sum_{j \in I} j C_{T}^{(T+j) / 2} / 2^{T-1} \\
\mathbf{D}\left|\eta_{i}\right|=\sum_{j \in I} j^{2} C_{T}^{(T+j) / 2} / 2^{T-1}-\left(\mathbf{E}\left|\eta_{i}\right|\right)^{2} \\
\mathbf{E}\left|\eta_{i}\right|^{3}=\sum_{j \in I} j^{3} C_{T}^{(T+j) / 2} / 2^{T-1}, \\
\left.\mathbf{E}|| \eta_{i}|-\mathbf{E}| \eta_{i}\right|^{3}=\sum_{k \in I, k \geq M\left|\eta_{i}\right|}\left(k-\mathbf{E}\left|\eta_{i}\right|\right)^{3} C_{T}^{(T+k) / 2} / 2^{T-1}+ \\
\sum_{1 \leq k<M\left|\eta_{i}\right|, k \in I}\left(\mathbf{E}\left|\eta_{i}\right|-k\right)^{3} C_{T}^{(T+k) / 2} / 2^{T-1}=\mathbf{E}\left(\left|\eta_{i}\right|-\mathbf{E}\left|\eta_{i}\right|\right)^{3}+ \\
+2 \sum_{1 \leq k<M\left|\eta_{i}\right|, k \in I}\left(\mathbf{E}\left|\eta_{i}\right|-k\right)^{3} .
\end{gathered}
$$

Величину

$$
\mathbf{E}\left|\left(\left|\eta_{i}\right|-\mathbf{E}\left|\eta_{i}\right|\right) / \sqrt{\mathbf{D}\left|\eta_{i}\right|}\right|^{3}
$$

при $T=t_{0 i}\left(t_{1 i}\right)$ обозначим $\mu_{0 i}\left(\mu_{1 i}\right)$.

Введём обозначения:

- $A_{i}$ есть событие, заключающееся в выполнении хотя бы одного из двух условий теоремы 1 ;

- $\quad B_{0 i}$ есть событие, при котором выполняются неравенства (4);

- $\quad B_{1 i}$ есть событие, при котором выполняются неравенства (5).

Напомним, что при осуществлении события $A_{i}$ система неравенств не имеет решений, а при осуществлении события $B_{1 i}\left(B_{0 i}\right)$ находится значение $x_{i}=1(0)$.

Теорема 2. Пусть для некоторого $i \in\{1,2, \ldots, n\}$ известны $n_{1 i}, n_{2 i}, n_{3 i}$ и нечётные числа $t_{0 i}, t_{1 i}$. Тогда справедливы следующие оченки:

$$
\begin{gathered}
\mathbf{P}\left\{A_{i}\right\}=\alpha_{0}+\alpha_{1} \cdot\left(1-\alpha_{0}-\beta\right)+\varepsilon_{1}, \\
\mathbf{P}\left\{B_{1 i}\right\}=\alpha_{1} \beta+\varepsilon_{2}, \\
\mathbf{P}\left\{B_{0 i}\right\}=\left(1-\alpha_{1}\right)\left(1-\alpha_{0}-\beta\right)+\varepsilon_{3}, \\
\mathbf{P}\left\{\overline{A_{i} \cup B_{1 i} \cup B_{0 i}}\right\}=\left(1-\alpha_{1}\right) \beta+\varepsilon_{4},
\end{gathered}
$$


где

$$
\begin{gathered}
\alpha_{0}=\Phi\left(\frac{1}{\sigma_{i}} \sqrt{\frac{t_{0 i}}{\left(n_{1 i}+n_{3 i}\right)}}-\frac{m_{i}}{\sigma_{i}} \sqrt{\left(n_{1 i}+n_{3 i}\right)}\right), \\
\alpha_{1}=\Phi\left(\frac{1}{\sigma_{i}} \sqrt{\frac{t_{1 i}}{\left(n_{1 i}+n_{2 i}\right)}}-\frac{m_{i}}{\sigma_{i}} \sqrt{\left(n_{1 i}+n_{2 i}\right)}\right), \\
\beta=1-\Phi\left(\frac{1}{\sigma_{i}} \sqrt{\frac{2 t_{0 i}}{\left(n_{1 i}+n_{3 i}\right)}}-\frac{m_{i}}{\sigma_{i}} \sqrt{\left(n_{1 i}+n_{3 i}\right)}\right), \\
\Phi(x)=\frac{1}{\sqrt{2 \pi}} \int_{-\infty}^{x} e^{-z^{2} / 2} d z .
\end{gathered}
$$

Величины $\varepsilon_{1}, \varepsilon_{2}, \varepsilon_{3}, \varepsilon_{4}$ удовлетворяют следуюшим соотношениям:

$$
\begin{gathered}
\varepsilon_{1}+\varepsilon_{2}+\varepsilon_{3}+\varepsilon_{4}=0, \quad\left|\varepsilon_{1}\right| \leq C_{0}+C_{1}, \quad\left|\varepsilon_{2}\right| \leq \alpha_{1} C_{0}+C_{1}, \\
\left|\varepsilon_{3}\right| \leq 2\left(1-\alpha_{0}\right) C_{0}+C_{1}, \quad\left|\varepsilon_{4}\right| \leq\left(1-\alpha_{1}\right) C_{0}+C_{1}, \\
C_{0}=C \mu_{0 i} / \sqrt{\left(n_{1 i}+n_{3 i}\right)}, \quad C_{1}=C \mu_{1 i} / \sqrt{\left(n_{1 i}+n_{2 i}\right)}, \quad C \leq 0,79,
\end{gathered}
$$

конкретные значения $m_{i 0}, m_{i 1}, \sigma_{i 0}, \sigma_{i 1}, \mu_{0 i}, \mu_{1 i}$ находятся по формулам (6), (7), (8), (9).

Доказательство. Рассмотрим вероятность

$$
\mathbf{P}\left\{v_{1 i}+v_{3 i}<t_{0 i}\right\} \text {. }
$$

Случайные величины $v_{1 i}\left(v_{3 i}\right)$ есть суммы $n_{1 i}\left(n_{3 i}\right)$ независимых случайных величин, имеющих такое же распределение, как и случайная величина

$$
\left|\eta_{i}\right|=\left|\sum_{j=1}^{T} \xi_{j i}\right|
$$

при $T=t_{0 i}$. Поэтому для функции распределения $F(y)$ случайной величины

$$
\frac{v_{1 i}+v_{3 i}-\mathbf{E}\left(v_{1 i}+v_{3 i}\right)}{\sqrt{\mathbf{D}\left(v_{1 i}+v_{3 i}\right)}}
$$

согласно теореме Берри-Эссена (см. [2], стр. 46) имеет место неравенство

$$
\max _{y}|F(y)-\Phi(y)| \leq C \mathbf{E}\left|\frac{\left|\eta_{i}\right|-\mathbf{E}\left|\eta_{i}\right|}{\sqrt{\mathbf{D}\left|\eta_{i}\right|}}\right|^{3} / \sqrt{\left(n_{1 i}+n_{3 i}\right)}, \quad C \leq 0,79 .
$$

Следовательно,

$$
\begin{gathered}
\mathbf{P}\left\{v_{1 i}+v_{3 i}<t_{0 i}\right\}= \\
\left.=\frac{v_{1 i}+v_{3 i}-m_{i}\left(n_{1 i}+n_{3 i}\right) \sqrt{t_{0 i}}}{\sigma_{i} \sqrt{t_{0 i}\left(n_{1 i}+n_{3 i}\right)}}<\frac{1}{\sigma_{i}}\left[\sqrt{\frac{t_{0 i}}{n_{1 i}+n_{3 i}}}-m_{i} \sqrt{\left(n_{1 i}+n_{3 i}\right)}\right]\right\}=\alpha_{0}+\delta_{0},
\end{gathered}
$$




$$
\delta_{0} \leq C \cdot \mu_{0 i} / \sqrt{\left(n_{1 i}+n_{3 i}\right)}=C_{0} .
$$

Аналогично находим

$$
\mathbf{P}\left\{\eta_{1 i}+\eta_{2 i}<t_{1 i}\right\}=\alpha_{1}+\delta_{1}, \quad \delta_{1}<C \cdot \mu_{1 i} / \sqrt{\left(n_{1 i}+n_{2 i}\right)}=C_{1} .
$$

Вероятности событий $\left\{v_{1 i}+v_{3 i} \geq 2 t_{0 i}\right\},\left\{\eta_{1 i}+\eta_{2 i} \geq t_{1 i}\right\},\left\{t_{0 i} \leq v_{1 i}+v_{3 i}<2 \cdot t_{0 i}\right\}$ оцениваются следующим образом:

$$
\begin{gathered}
\mathbf{P}\left\{v_{1 i}+v_{3 i} \geq 2 t_{0 i}\right\}=1-\mathbf{P}\left\{v_{1 i}+v_{3 i}<2 t_{0 i}\right\}=\beta+\delta_{2}, \quad \delta_{2} \leq C_{0}, \\
\mathbf{P}\left\{\eta_{1 i}+\eta_{2 i} \geq t_{1 i}\right\}=1-\mathbf{P}\left\{\eta_{1 i}+\eta_{2 i}<t_{1 i}\right\}=1-\alpha_{1}-\delta_{1}, \\
\mathbf{P}\left\{t_{0 i} \leq v_{1 i}+v_{3 i}<2 t_{0 i}\right\}=1-\beta-\delta_{2}-\alpha_{0}-\delta_{0} .
\end{gathered}
$$

Таким образом, находим

$$
\begin{gathered}
\mathcal{E}_{1}=\delta_{0}\left(1-\alpha_{1}\right)-\alpha_{1} \delta_{2}+\delta_{1}\left(1-\alpha_{0}-\beta-\delta_{0}-\delta_{2}\right), \quad\left|\varepsilon_{1}\right| \leq C_{0}+C_{1}, \\
\varepsilon_{2}=\alpha_{1} \delta_{2}+\beta \delta_{1}+\delta_{1} \delta_{2}, \quad\left|\varepsilon_{2}\right| \leq \alpha_{1} C_{0}+C_{1}, \\
\varepsilon_{3}=\delta_{1}\left(1-\alpha_{0}-\beta-\delta_{0}-\delta_{2}\right)-\left(\delta_{0}+\delta_{2}\right) \cdot\left(1-\alpha_{0}\right), \quad\left|\varepsilon_{3}\right| \leq C_{1}+2\left(1-\alpha_{0}\right) \cdot C_{0}, \\
\varepsilon_{4}=\delta_{2}\left(1-\alpha_{1}\right)-\delta_{1}\left(\beta+\delta_{2}\right), \quad\left|\varepsilon_{4}\right| \leq C_{1}+\left(1-\alpha_{1}\right) \cdot C_{0} .
\end{gathered}
$$

Принимая во внимание независимость рассматриваемых случайных величин и неравенства (10), приходим к утверждению теоремы.

\section{3. Заведомо совместная система неравенств}

Пусть исходной системе линейных псевдобулевых неравенств (1) при любом значении числа неравенств $t$ удовлетворяет вектор $x^{0}=$ $=\left(x_{1}^{0}, x_{2}^{0}, \ldots, x_{n}^{0}\right)$. В этом случае скалярное произведение

$$
\left(\overline{a_{i}}, x^{0}\right) \geq b_{i}, \quad \bar{a}_{i}=\left(a_{i 1}, a_{i 2}, \ldots, a_{i n}\right), \quad i=1,2, \ldots .
$$

Многие результаты, полученные в п. 2 , переносятся и на этот случай. В частности, результаты по нахождению значений отдельных неизвестных (неравенства (4), (5)).

Как и в п. 2, рассмотрим пример, в котором коэффициенты в неравенствах независимы и принимают только два значения 1 и -1 с равной вероятностью. Считаем, что множество возможных решений $X$ состоит из векторов фиксированного нечётного веса, $x^{0} \in X,\left|x^{0}\right|=2 k+1, b_{i}=1, i=1,2, \ldots, t$.

Лемма 1. Если $x_{1}^{0}=1$, то

$$
\begin{aligned}
& \mathbf{P}\left\{\left(\overline{a_{i}}, x^{0}\right) \geq 1 \quad \text { । } \quad a_{i 1}=1\right\}=\mathbf{P}\left\{\left(\overline{a_{i}}, x^{0}\right) \leq-1 \quad \text { । } \quad a_{i 1}=-1\right\}=\frac{1}{2}+\frac{C_{2 k}^{k}}{2^{2 k+1}}, \\
& \mathbf{P}\left\{\left(\overline{a_{i}}, x^{0}\right) \geq 1 \quad \text { । } \quad a_{i 1}=-1\right\}=\mathbf{P}\left\{\left(\overline{a_{i}}, x^{0}\right) \leq-1 \quad \text { । } \quad a_{i 1}=1\right\}=\frac{1}{2}\left(1-\frac{C_{2 k}^{k}}{2^{2 k}}\right) .
\end{aligned}
$$


Для $x_{1}^{0}=0$ имеют место равенства

$$
\begin{gathered}
\mathbf{P}\left\{\left(\overline{a_{i}}, x^{0}\right) \geq 1 \quad \text { । } \quad a_{i 1}=1\right\}=\mathbf{P}\left\{\left(\overline{a_{i}}, x^{0}\right) \leq-1 \quad \text { । } \quad a_{i 1}=-1\right\}=\frac{1}{2}, \\
\mathbf{P}\left\{\left(\overline{a_{i}}, x^{0}\right) \geq 1 \quad \text { । } \quad a_{i 1}=-1\right\}=\mathbf{P}\left\{\left(\overline{a_{i}}, x^{0}\right) \leq-1 \quad \text { । } \quad a_{i 1}=1\right\}=\frac{1}{2}, \quad i=1,2, \ldots, t .
\end{gathered}
$$

Доказательство. Второе утверждение очевидно, а первое утверждение следует из формул

$$
\begin{gathered}
\mathbf{P}\left\{\left(\overline{a_{i}}, x^{0}\right) \geq 1 \mid a_{i 1}=1\right\}=\mathbf{P}\left\{a_{i 2} x_{2}^{0}+\ldots+a_{i n} x_{n}^{0} \geq 0\right\}=\frac{1}{2^{2 k}} \cdot \sum_{j \geq 0} C_{2 k}^{k+j}=\frac{1}{2}\left(1+\frac{C_{2 k}^{k}}{2^{2 k}}\right), \\
\mathbf{P}\left\{\left(\overline{a_{i}}, x^{0}\right) \geq 1 \mid a_{i 1}=-1\right\}=\mathbf{P}\left\{a_{i 2} x_{2}^{0}+\ldots+a_{i n} x_{n}^{0} \geq 2\right\}=\frac{1}{2^{2 k}} \cdot \sum_{j \geq 1} C_{2 k}^{k+j}=\frac{1}{2}\left(1-\frac{C_{2 k}^{k}}{2^{2 k}}\right) .
\end{gathered}
$$

Очевидны следующие утверждения.

Лемма 2. При $x_{j}^{0}=1$ случайная величина

$$
t_{1 j}=\left|\left\{i: a_{i j}=1, i \in\{1,2, \ldots, t\}\right\}\right|
$$

имеет биномиальное распределение с параметрами $\left(t, \frac{1}{2}+C_{2 k}^{k} / 2^{2 k+1}\right)$, а случайная величина

$$
t_{0 j}=\left|\left\{i: a_{i j}=-1, i \in\{1,2, \ldots, t\}\right\}\right|
$$

распределена по биномиальному закону с параметрами $\left(t, \frac{1}{2}-C_{2 k}^{k} / 2^{2 k+1}\right)$.

Для $x_{j}^{0}=0$ обе случайные величины имеют одинаковое биномиальное распределение с параметрами $(t, 1 / 2)$. Во всех случаях $t_{0 j}+t_{1 j}=t$ для любого $j \in\{1,2, \ldots, n\}$.

ЗАМЕЧАНИЕ. По существу, утверждения лемм 1,2 позволяют строить критерий отличия случайной системы неравенств от заведомо совместной случайной системы неравенств.

Лемма 3. Пусть известны $t_{1 j}, j \in\{1,2, \ldots, n\}$. Тогда апостериорная вероятность

$$
\begin{aligned}
\overline{p_{j}}=\mathbf{P}\left\{x_{j}^{0}=1 \mid t_{1 j}, t\right\}= & {\left[1+\frac{n-2 k-1}{2 k+1}\left(1-\left(\frac{C_{2 k}^{k}}{2^{2 k}}\right)^{2}\right)^{-t / 2}\left(\frac{2^{2 k}-C_{2 k}^{k}}{2^{2 k}+C_{2 k}^{k}}\right)^{\zeta_{j}}\right]^{-1}, } \\
& \zeta_{j}=t_{1 j}-t / 2, \quad j \in\{1,2, \ldots, n\} .
\end{aligned}
$$

Доказательство. Так как априорная вероятность

$$
p=\mathbf{P}\left\{x_{j}^{0}=1\right\}=(2 k+1) / n, \quad j \in\{1,2, \ldots, n\},
$$


то, используя леммы 1,2 и идею, предложенную в [3], находим

$$
\overline{p_{j}}=\frac{p p_{1}^{t_{1 j}}\left(1-p_{1}\right)^{t-t_{1 j}}}{p p_{1}^{t_{1 j}}\left(1-p_{1}\right)^{t-t_{1 j}}+(1-p) 2^{-t}}, \quad p_{1}=\frac{1}{2}\left(1+\frac{C_{2 k}^{k}}{2^{2 k}}\right) .
$$

Поделим числитель и знаменатель этой дроби на числитель и придём к выражению (11).

\section{Следствие 1. Апостериорная вероятность}

$$
\mathbf{P}\left\{x_{j}^{0}=0 \mid t_{1 j}, t\right\}=1-\overline{p_{j}}=\left[1+\frac{2 k+1}{n-2 k-1}\left(1-\left(\frac{C_{2 k}^{k}}{2^{2 k}}\right)^{2}\right)^{t / 2}\left(\frac{2^{2 k}-C_{2 k}^{k}}{2^{2 k}+C_{2 k}^{k}}\right)^{-\zeta_{j}}\right]^{-1} .
$$

Для иллюстрации утверждений лемм 1-3 приведем конкретный пример. Пусть $n=6, x_{1}^{0}=x_{2}^{0}=x_{3}^{0}=1, x_{4}^{0}=x_{5}^{0}=x_{6}^{0}=0$. При наших предположениях о коэффициентах существует 32 неравенства вида $a_{1} x_{1}+\ldots+a_{6} x_{6} \geq 1$ и 32 неравенства вида $b_{1} x_{1}+\ldots+b_{6} x_{6} \leq-1$. Найдем число неравенств с $a_{1}=1$ и $b_{1}=-1$. В нашем случае эти неравенства выглядят следующим образом:

$$
a_{2}+a_{3} \geq 0, \quad b_{2}+b_{3} \leq 0 .
$$

Число таких неравенств для каждого вида равно $2^{3}(1+2)=24$. Следовательно, вероятность появления в заведомо совместной системе (1) неравенства с $a_{1}=1$ равна $48 / 64=3 / 4$ и число таких неравенств имеет биномиальное распределение с параметрами $(t, 3 / 4)$.

Пусть $t=8, t_{11}=7$. Тогда

$$
\bar{p}_{1}=\mathbf{P}\left(x_{1}^{0}=1 \mid t_{11}=7, t=8\right)=\left[1+\left(\frac{3}{4}\right)^{4} \frac{1}{3}\right]^{-1} \approx 0,9 .
$$

Для случайной системы неравенств $\mathbf{E} t_{11}=4$, а вероятность события $\left\{t_{11}=7\right\}$ равна $C_{8}^{7}\left(\frac{1}{2}\right)^{8}=\frac{1}{32}$.

Пусть $H_{0 j}\left(H_{1 j}\right)$ обозначает гипотезу, по которой $x_{j}^{0}=0(1)$, а $I_{0 j}\left(I_{1 j}\right)-$ индикатор этой гипотезы, $j \in\{1,2, \ldots, n\}$. Гипотезу $H_{0 j}\left(H_{1 j}\right)$ считаем справедливой, если $\varsigma_{j}<Q>0\left(\varsigma_{j} \geq Q\right)$. При $n \rightarrow \infty$ найдем такие $k(n), t(n), Q(n)$, для которых

$$
\sum_{j \in\{1, \ldots, n\}: x_{j}^{0}=0} I_{0 j} \sim n-2 k-1, \quad \sum_{j \in\{1, \ldots, n\}: x_{j}^{0}=1} I_{1 j} \sim 2 k+1,
$$

и суммарная ошибка стремится к нулю. 
Tеорема 3. Пусть $n \rightarrow \infty, t=2 Q \sqrt{\pi \theta n}+O(1)=o\left(n^{2}\right), \theta=(2 k+1) / n+O\left(\frac{1}{n}\right)$, $0<\varepsilon<\theta<1-\varepsilon$. Тогда среднее число неправильно принятых гипотез $H_{0 j}\left(H_{1 j}\right), j \in\{1,2, \ldots, n\}$, эквивалентно величине $(1-\theta) n \beta(\theta n \alpha)$,

$$
\alpha \sim \beta \sim \frac{1}{\sqrt{2 \pi}} \int_{t_{\alpha}}^{\infty} e^{-z^{2} / 2} d z, t_{\alpha}=\sqrt{\frac{t}{\pi \theta n}}\left[1+O\left(\frac{1}{n}\right)\right] .
$$

Доказательство. Согласно утверждению леммы 2

$$
\begin{gathered}
\mathbf{E}_{0} \zeta_{j}=\mathbf{E}_{0}\left(t_{1 j}-\frac{t}{2}\right)=0, \mathbf{D}_{0} \zeta_{j}=\frac{t}{4}, \\
\mathbf{E}_{1} \zeta_{j}=\frac{t}{2} C_{2 k}^{k} / 2^{2 k}, \quad \mathbf{D}_{1} \zeta_{j}=\frac{t}{4}\left[1-\left(\frac{C_{2 k}^{k}}{2^{2 k}}\right)^{2}\right], \quad j=1,2, \ldots, n .
\end{gathered}
$$

Далее находим

$$
\begin{gathered}
\left(\mathbf{E}_{1} \zeta_{j}-\mathbf{E}_{0} \zeta_{j}\right) / 2=\frac{t}{4} \sqrt{\frac{1}{\pi \theta n}}\left(1+O\left(\frac{1}{n}\right)\right)=\frac{Q}{2}\left[1+O\left(\frac{1}{n}\right)\right], \\
t_{\alpha}=\frac{Q}{2}\left[1+O\left(\frac{1}{n}\right)\right] / \sqrt{\frac{t}{4}}=\sqrt{\frac{t}{\pi \theta n}}\left[1+O\left(\frac{1}{n}\right)\right], \\
t_{\beta}-t_{\alpha}=O\left(\sqrt{\frac{t}{\pi \theta n^{3}}}\right)=o\left(\frac{1}{\sqrt{n}}\right) .
\end{gathered}
$$

Этим заканчивается доказательство теоремы.

Следствие 2. Если $t=2 \pi \theta n \ln n+O(1)$, то для всех $j=1,2, \ldots, n$ будет принята правильная гипотеза с вероятностью, стремящейся к единице при $n \rightarrow \infty$.

Доказательство. Согласно теореме 3 в этом случае

$$
t_{\alpha}=\sqrt{2 \ln n}\left[1+O\left(\frac{1}{\sqrt{n}}\right)\right],
$$

поэтому

$$
\alpha \sim \frac{1}{\sqrt{2 \pi}} \int_{t_{\alpha}}^{\infty} e^{-z^{2} / 2} d z \sim \frac{1}{t_{\alpha} \sqrt{2 \pi}} e^{-t_{\alpha}^{2} / 2} \sim \frac{1}{n \sqrt{4 \pi \ln n}}, \quad n \alpha \rightarrow 0 .
$$

В условиях теоремы 3 найдём асимптотическое представление формулы (11). Очевидны следующие асимптотические выкладки:

$$
\frac{n-2 k-1}{2 k+1}=\frac{1-\theta}{\theta}+O\left(\frac{1}{n}\right), \quad \Delta=\frac{C_{2 k}^{k}}{2^{2 k}}=\sqrt{\frac{1}{\pi \theta n}}\left(1+O\left(\frac{1}{n}\right)\right),
$$




$$
\begin{gathered}
\left(1-\Delta^{2}\right)^{-t / 2}=\exp \left\{\frac{t}{2} \cdot\left(\Delta^{2}+\frac{\Delta^{4}}{2}+\ldots\right)\right\}=\exp \left\{\frac{t}{2 \pi \theta n}\left(1+O\left(\frac{1}{n}\right)\right)\right\}, \\
\left(\frac{1-\Delta}{1+\Delta}\right)^{\varsigma_{j}}=\exp \left\{-2 \zeta_{j}\left(\Delta+\frac{\Delta^{3}}{3}+\ldots\right)\right\}=\exp \left\{-2 \zeta_{j} \sqrt{\frac{1}{\pi \theta n}}\left(1+O\left(\frac{1}{n}\right)\right)\right\} .
\end{gathered}
$$

Таким образом,

$$
\begin{aligned}
\overline{p_{j}} & =\left[1+\frac{1-\theta}{\theta} \exp \left\{\frac{Q}{\sqrt{\pi \theta n}}\left[1+O\left(\frac{1}{n}\right)\right]-2 \zeta_{j} \sqrt{\frac{1}{\pi \theta n}}\left[1+O\left(\frac{1}{n}\right)\right]\right\}\right]^{-1}, \\
1-\overline{p_{j}} & =\left[1+\frac{\theta}{1-\theta} \exp \left\{-\frac{Q}{\sqrt{\pi \theta n}}\left[1+O\left(\frac{1}{n}\right)\right]+2 \zeta_{j} \sqrt{\frac{1}{\pi \theta n}}\left[1+O\left(\frac{1}{n}\right)\right]\right\}\right]^{-1} .
\end{aligned}
$$

Если верна гипотеза $H_{1 j}$, то в худшем случае $\zeta=Q+O(1)$

и

$$
1-\overline{p_{j}} \sim \frac{1-\theta}{\theta} \exp \left\{-\frac{t}{2 \pi \theta n}\right\} .
$$

Это означает, что $\bar{p}_{j} \rightarrow 1$ при $t / n \rightarrow \infty$.

\section{4. Неравенства с искажениями}

Приведём три возможных вида искажений для рассмотренного в п. 3 примера.

1) Знаки неравенства и правые части меняются на противоположные с вероятностью $p<1 / 2$, т. е. с вероятностью $p$ истинное решение $x^{0}$ не удовлетворяет новому неравенству. Найдём вероятность ошибки для суммы двух неравенств

$$
\left(\overline{a_{i}}, x\right) \geq 1, \quad\left(\overline{a_{j}}, x\right) \geq 1 .
$$

Лемма 4. Вероятность того, что истинное решение $x^{0}$ не удовлетворяет неравенству

$$
\left(\overline{a_{i}}+\overline{a_{j}}, x^{0}\right) \geq 2 \text {, }
$$

равна выражению

$$
\mathbf{P}\left\{\left(\overline{a_{i}}+\overline{a_{j}}, x^{0}\right) \leq 0\right\}=p\left[1+(1-p) C_{4 k+2}^{2 k+1} / 2^{4 k+1}\right] .
$$


Доказательство. По формуле полной вероятности находим

$$
\begin{gathered}
\mathbf{P}\left\{\left(\overline{a_{i}}+\overline{a_{j}}, x^{0}\right) \leq 0\right\}= \\
=(1-p)^{2} \cdot 0+p^{2} \cdot 1+2 p(1-p) \mathbf{P}\left\{\left(\overline{a_{i}}+\overline{a_{j}}, x^{0}\right) \leq 0 ।\left(\overline{a_{i}}, x^{0}\right) \leq-1,\left(\overline{a_{j}}, x^{0}\right) \geq 1\right\} .
\end{gathered}
$$

Далее, учитывая симметрию в распределениях рассматриваемых случайных величин, приходим к формулам

$$
\begin{gathered}
\mathbf{P}\left\{\left(\overline{a_{i}}+\overline{a_{j}}, x^{0}\right) \leq 0 ।\left(\overline{a_{i}}, x^{0}\right) \leq-1,\left(\overline{a_{j}}, x^{0}\right) \geq 1\right\}= \\
=\mathbf{P}\left\{\left(\overline{a_{i}}, x^{0}\right) \geq\left(\overline{a_{j}}, x^{0}\right) ।\left(\overline{a_{i}}, x^{0}\right) \geq 1,\left(\overline{a_{j}}, x^{0}\right) \geq 1\right\}= \\
=4 \sum_{l \geq 1} \mathbf{P}\left\{\left(\overline{a_{j}}, x^{0}\right)=l\right\} \sum_{h \geq l} \mathbf{P}\left\{\left(\overline{a_{i}}, x^{0}\right)=h\right\}= \\
=2\left[\frac{1}{2} \sum_{l \geq 1}\left(\mathbf{P}\left\{\left(\overline{a_{j}}, x^{0}\right)=l\right\}\right)^{2}+\frac{1}{2}\left(\sum_{l \geq 1} \mathbf{P}\left\{\left(\overline{a_{j}}, x^{0}\right)=l\right\}\right)^{2}\right] .
\end{gathered}
$$

Так как $\left|x^{0}\right|=2 k+1$, то

$$
\begin{gathered}
\mathbf{P}\left\{\left(\overline{a_{j}}, x^{0}\right)=l\right\}=C_{2 k+1}^{(2 k+1+l) / 2} / 2^{2 k+1}, \quad l=1,3, \ldots, 2 k+1, \\
\sum_{l \geq 1}\left(\mathbf{P}\left\{\left(\overline{a_{j}}, x^{0}\right)=l\right\}\right)^{2}=\sum_{h=1}^{k+1}\left(C_{2 k+1}^{k+h} / 2^{2 k+1}\right)^{2}=\frac{1}{2} C_{4 k+2}^{2 k+1} / 2^{4 k+2} .
\end{gathered}
$$

Следовательно,

$$
\begin{gathered}
\mathbf{P}\left\{\left(\overline{a_{i}}+\overline{a_{j}}, x^{0}\right) \leq 0\right\}=p\left[p+(1-p)\left(1+\frac{C_{4 k+2}^{2 k+1}}{2^{4 k+1}}\right)\right]= \\
=p\left[1+(1-p) \frac{C_{4 k+2}^{2 k+1}}{2^{4 k+1}}\right],
\end{gathered}
$$

что и требовалось доказать.

2) В правые части неравенств добавляем две единицы. Тем самым допускаем возможность в каждом неравенстве ошибки, но делаем более эффективным применение метода выделения и оценки отдельных неизвестных (см. п. 2).

Лемма 5. Вероятность того, что истинное решение $x^{0}$ не удовлетворяет неравенству

$$
\left(\bar{a}_{i}+\bar{a}_{j}, x^{0}\right) \geq 6
$$

равна выражению

$$
\mathbf{P}\left\{\left(\bar{a}_{i}+\bar{a}_{j}, x^{0}\right) \leq 4\right\}=C_{2 k+1}^{k}\left(C_{2 k+1}^{k}+2 C_{2 k+1}^{k-1}\right) / 2^{4 k} .
$$


Доказательство. Учитывая исходные неравенства

$$
\left(\bar{a}_{i}, x^{0}\right) \geq 1, \quad\left(\bar{a}_{j}, x^{0}\right) \geq 1,
$$

находим

$$
\begin{gathered}
\mathbf{P}\left\{\left(\bar{a}_{i}+\bar{a}_{j}, x^{0}\right) \leq 4\right\}=\mathbf{P}\left\{\left(\bar{a}_{i}, x^{0}\right)=1\right\}\left[\mathbf{P}\left\{\left(\bar{a}_{j}, x^{0}\right)=1\right\}+\mathbf{P}\left\{\left(\bar{a}_{j}, x^{0}\right)=3\right\}\right]+ \\
+\mathbf{P}\left\{\left(\bar{a}_{i}, x^{0}\right)=3\right\} \mathbf{P}\left\{\left(\bar{a}_{j}, x^{0}\right)=1\right\}= \\
=4 C_{2 k+1}^{k+1}\left(C_{2 k+1}^{k+1}+2 C_{2 k+1}^{k+2}\right) / 2^{4 k+2} .
\end{gathered}
$$

3) Возможны случаи, когда одновременно могут присутствовать два вида искажений. Для подсчёта $\mathbf{P}\left\{\left(\bar{a}_{i}+\bar{a}_{j}, x^{0}\right) \leq 4\right\}$ используем леммы 4,5 . С вероятностью $(1-p)^{2} \quad$ удовлетворялись неравенства $\left(\bar{a}_{i}, x^{0}\right) \geq 1$, $\left(\bar{a}_{j}, x^{0}\right) \geq 1, \quad$ с вероятностью $p^{2}$ исходными неравенствами были $\left(\bar{a}_{i}, x^{0}\right) \leq-1, \quad\left(\bar{a}_{j}, x^{0}\right) \leq-1$, а с одинаковой вероятностью $p(1-p)$ в системе присутствовали неравенства $\left(\bar{a}_{i}, x^{0}\right) \leq-1, \quad\left(\bar{a}_{j}, x^{0}\right) \geq 1$ или неравенства $\left(\bar{a}_{i}, x^{0}\right) \geq 1, \quad\left(\bar{a}_{j}, x^{0}\right) \leq-1$. С учётом этого находим

$$
\begin{gathered}
\mathbf{P}\left\{\left(\bar{a}_{i}+\bar{a}_{j}, x^{0}\right) \leq 4\right\}=(1-p)^{2} C_{2 k+1}^{k}\left(C_{2 k+1}^{k}+2 C_{2 k+1}^{k-1}\right) / 2^{4 k}+ \\
+2 p(1-p) \mathbf{P}\left\{\left(\bar{a}_{i}+\bar{a}_{j}, x^{0}\right) \leq 4 \mathrm{I}\left(\bar{a}_{i}, x^{0}\right) \leq-1,\left(\bar{a}_{j}, x^{0}\right) \geq 1\right\}+p^{2}, \\
\mathbf{P}\left\{\left(\bar{a}_{i}, x^{0}\right) \leq 4-\left(\bar{a}_{j}, x^{0}\right) \mathrm{I}\left(\bar{a}_{i}, x^{0}\right) \leq-1,\left(\bar{a}_{j}, x^{0}\right) \geq 1\right\}= \\
=4 \sum_{l \geq 1, h \geq 1, h \leq 4+l} \mathbf{P}\left\{\left(\bar{a}_{i}, x^{0}\right)=-l\right\} \cdot \mathbf{P}\left\{\left(\bar{a}_{j}, x^{0}\right)=h\right\} .
\end{gathered}
$$

\section{Список литературы}

1. Балакин Г. В. О возможности решения систем линейных целочисленных уравнений методом выделения и оценки отдельных неизвестных. Дискрет. матем., 1994, т. 6, в. 1, с. 116-126.

2. Вероятность и математическая статистика. - Энциклопедия. - Москва, 1999.

3. Балакин Г. В. О вероятностном подходе к решению систем уравнений с целочисленными неизвестными. - Дискрет. матем., 1995, т. 7, в. 1, c. $88-98$. 